\title{
Making Innovative Tattoo Ink Products with Improved Safety: Possible and Impossible Ingredients in Practical Usage
}

\author{
Michael Dirks \\ $\mathrm{H}-\mathrm{A}-\mathrm{N} \mathrm{GmbH}$, Esslingen, Germany
}

\begin{abstract}
Today's tattoo inks are no longer just simple solids in liquid suspension. Nowadays, these inks are high-tech dispersions made from finely spread pigments in a bindersolvent mixture. These so-called colour dispersions must follow the modern standards of tattooing, which are increasing every year. They must be rich in chromophoric pigments and yet fluid, they must not dry rapidly, and there should be no occurrence of any sedimentation, even during longer tattoo seasons. An innovative tattoo ink should enable long-lasting, brilliant tattoos without a negative impact on the artist's workflow and of course without endangering the consumer. The high standard in tattoos, regarding the motives and techniques, that is witnessed today could not be achieved by the artists without quality tools and modern tattoo ink. This article will give the reader a brief overview of the different ingredients of tattoo ink and of the function of binding agents and solvents in modern tattoo ink as well as describe
\end{abstract}

what additives are used to achieve the desired behaviour during application. Furthermore, the article will take a look into the pigments that are used in tattoo ink and show why certain pigments are not suited for tattoo ink. The differences, advantages and disadvantages of organic and inorganic pigments will be explained.

(c) 2015 S. Karger AG, Basel

\section{Tattoo Inks}

Broadly speaking, tattoo inks are a suspension of non-soluable pigments in a liquid. The liquid of tattoo ink consists of two components: a binder and a solvent. The fine-dispersed pigment mixture is stabilised by additives. Preservatives can be added to prevent microbiological spoilage. Figure 1 shows a schematic of the composition of the main ingredients of tattoo ink. 


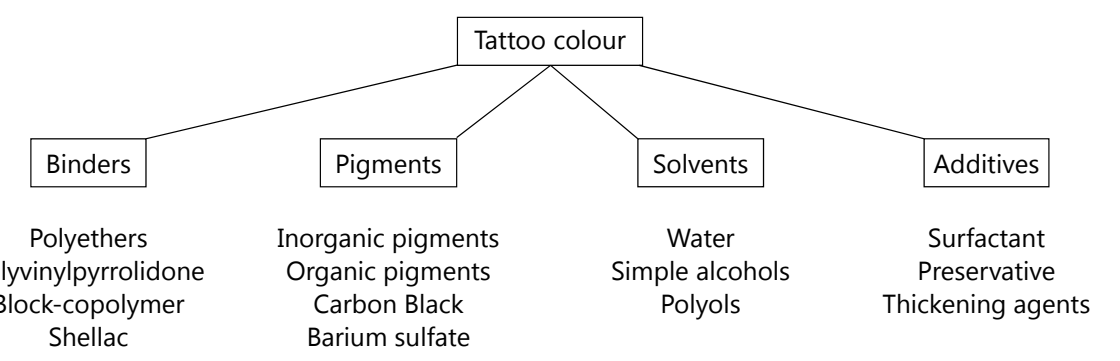

Fig. 1. Simplified representation of the composition of tattoo ink.

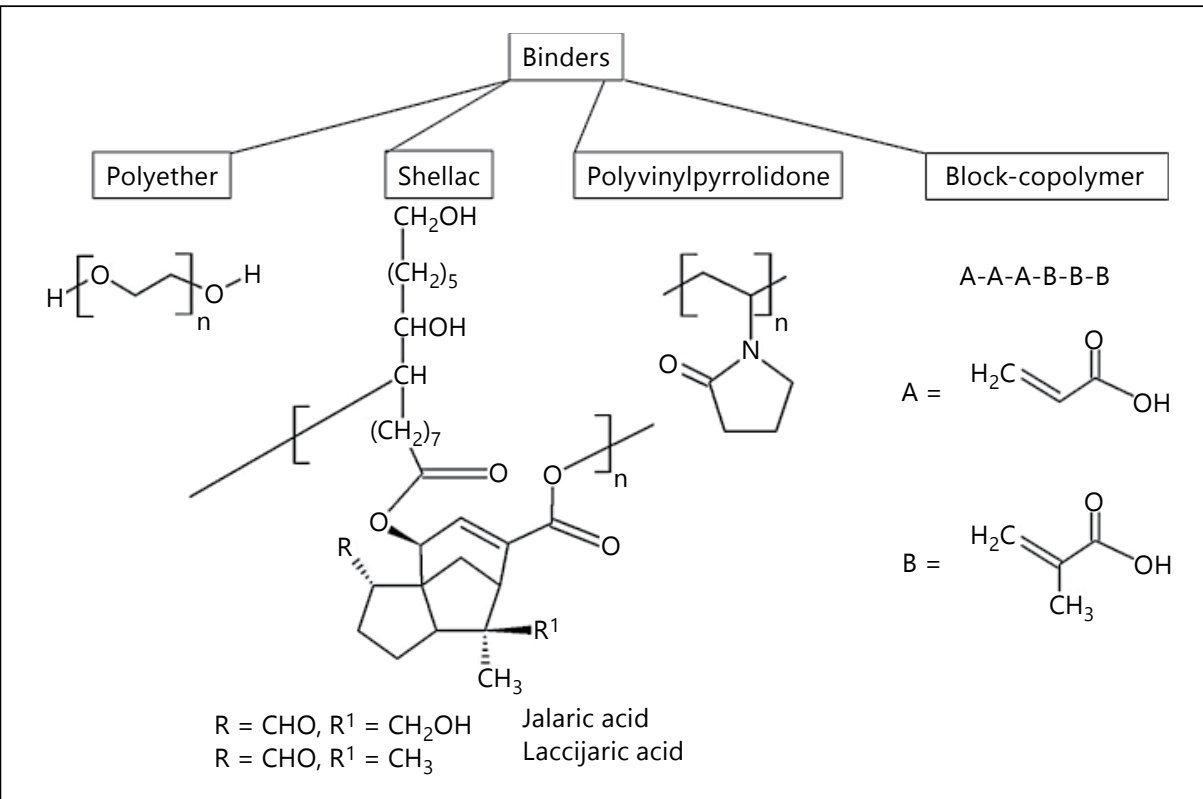

Fig. 2. Structural formulae of binding agents used in customary tattoo inks.

\section{Binding Agents}

Binding agents, or binders, are the non-volatile compounds of tattoo ink, not including pigments and filler but including additives [1]. The binder binds the pigment particles to each other and to the tattooing needle for easier injection of the ink into the skin. Figure 2 shows the most important binding agents in customary tattoo inks. Because of their high molar mass, the binding agents are limited in their bio-availability and are not seen as an acute hazard. The average molar masses can be found in table 1 .

\section{Solvents}

All of the above-mentioned binders are soluble in water or are neutralised with an alkaline solution. Because of this, water is the main ingredient of 
Table 1. Average molar masses of binding agents

\begin{tabular}{lc}
\hline Polymer & Molar mass \\
\hline Polyethylene glycol & $200-600 \mathrm{~g} \cdot \mathrm{mol}^{-1}$ (liquid); $>4,000 \mathrm{~g} \cdot \mathrm{mol}^{-1}$ (solid) \\
Poloxamer 188 & $7,680-9,510 \mathrm{~g} \cdot \mathrm{mol}^{-1}($ solid) \\
Polyvinylpyrrolidone & $2,500-2,500,000 \mathrm{~g} \cdot \mathrm{mol}^{-1}$ (solid) \\
Shellac & $1,500-2,500 \mathrm{~g} \cdot \mathrm{mol}^{-1}$ (solid) \\
Acrylate & $10,000-200,000 \mathrm{~g} \cdot \mathrm{mol}^{-1}$ (solid) \\
\hline
\end{tabular}

Table 2. Purposes of solvents used in tattoo inks

\begin{tabular}{ll}
\hline Solvent & Purpose \\
\hline Water & Solvation of binder \\
Ethanol & Regulates drying properties, masks smells \\
Isopropyl alcohol & Regulates drying properties, masks smells \\
Glycerin & Increasing viscosity, humectant \\
Propylene glycol & Humectant, increases dispersability \\
\hline
\end{tabular}

tattoo ink. Also, simple and polyvalent alcohols are used to define characteristics like drying properties, viscosity and dispersibility. The characteristics of solvents used in tattoo inks are shown in table 2 . With the exception of water, the usage of solvents should be limited because high concentrations of alcohol can irritate the skin.

\section{Additives}

Additives are auxiliary materials that are added to exclude unwanted characteristics or to enhance wanted characteristics [2]. The concentration of additives used in tattoo inks is not higher than 5\%. The used additives include the following: surfactants to adjust the surface as well as thickening and thixotroping agents and preservatives.

\section{Surface-Active Additives (Tensides)}

Tensides are used in tattoo inks to better disperse and stabilize the pigments during the production process, leading to a longer shelf life.
The power of attraction of the pigment molecules leads to cohesion, and therefore, they drive to have the smallest possible surface in a given volume. This property is known as surface tension and can lead to problems regarding interactions with other ingredients of the formulation. Fine-dispersed pigments tend to agglomerate to bigger structures and resist wetting by the binder solution if the surface tension of the substance is not adjusted with boundary layers. Compatibilisers, like tensides, are necessary for the complete removal of air from the agglomerates and complete coating of the pigment particles with the binder solution.

\section{Thickening Agents}

The only way to inhibit the reagglomeration and therefore sedimentation of inks during longer storage is to enhance its structural viscosity and thixotropy. Thixotroping agents, like silica, build up the yield point without impairing the shear conditions during processing. Figure 3 shows, in a schematic way, that the edges of silica are flat and 
Fig. 3. Card house-like structures of silica.
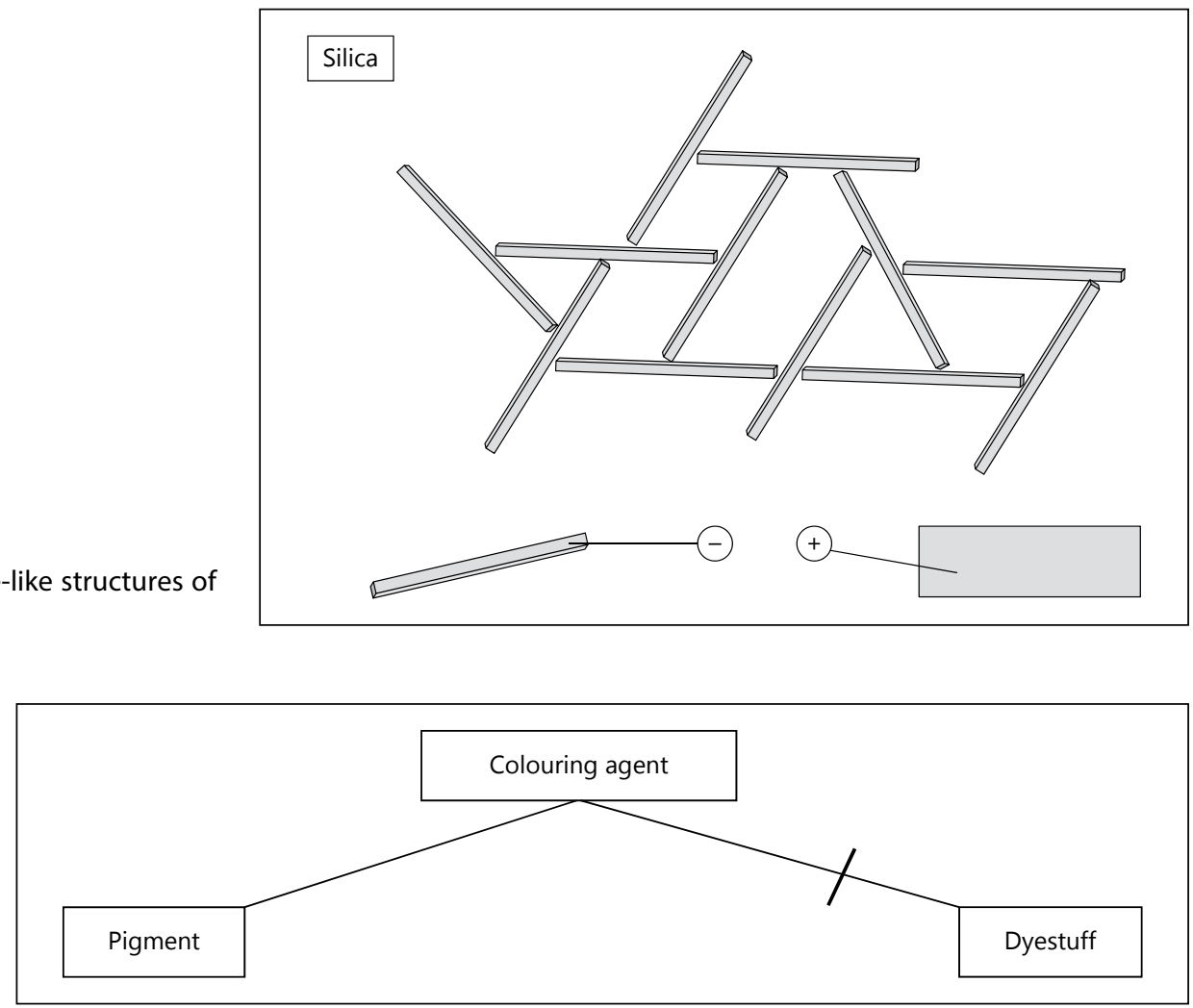

Fig. 4. Per definition, dyes and pigments are colorants.

that the molecules are arranged in a card houselike structure in idle conditions because of different surface charges.

\section{Preservatives}

As products with a high portion of water, tattoo inks must be protected against microbiological spoilage. To reduce the risk of allergic reaction, it's best practise to waive preservatives. Waterrich media provide better reproduction opportunities than water-scarce media. The water activity, as a unit for the availability of water, and not the portion of water in a medium is crucial for the reproduction of microorganisms. To deny microorganisms the opportunity to reproduce in tattoo ink, the water activity value should be $<0.6$ [3].

\section{Pigments}

Pigments are by definition insoluble, organic or inorganic colorants in medium, and dyes are soluble organic colorants in medium [4]. Dyes are not suitable as tattoo ink because they are soluble and will be biodegraded very fast after application. Figure 4 shows the divison of colouring agents into pigments and dyestuff. Figure 5 shows a rough division of the pigments and fillers used in tattoo inks. Every class of pigments has its advantages and disadvantages and have typical pigment impurities, i.e., inorganic impurities like heavy metals in metal oxide pigments, organic impurities like aromatic amines in organic coloured pigments, and impurities like polycyclic aromatic hydrocarbons (PAHs) in Carbon Black pigments. 


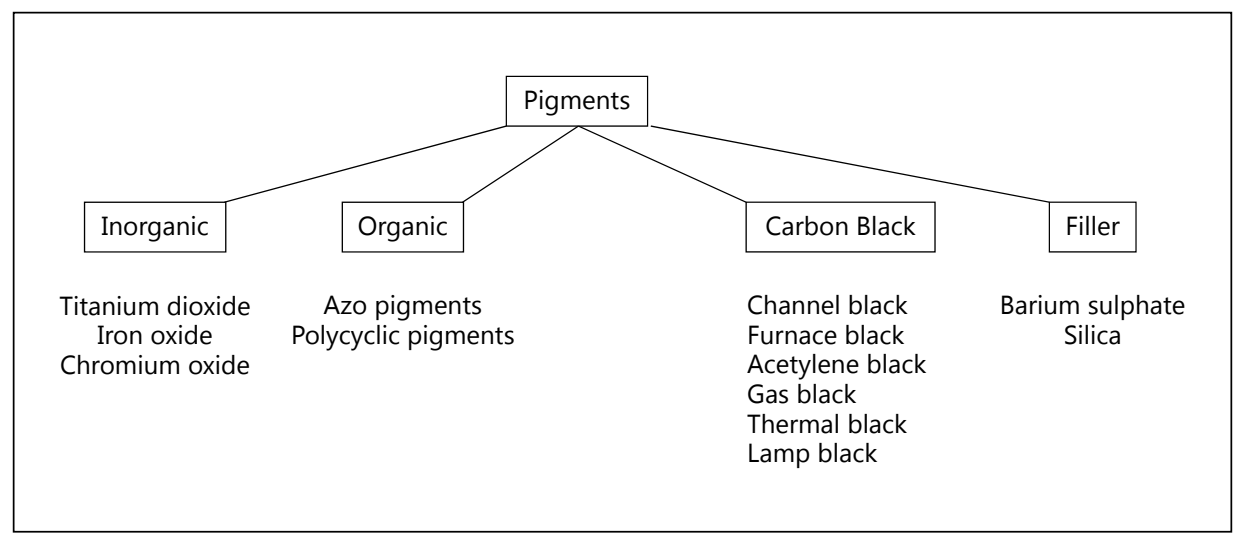

Fig. 5. Division of pigments.

\section{Inorganic Pigments}

The most-used inorganic pigments in tattoo inks are limited due to their application properties. The used pigments include iron oxide, titanium dioxide and chromium oxide green.

\section{Titanium Dioxide Pigments}

Titanium dioxide pigments show very little absorption ability and reflect almost unchanged white light because of its high scattering power. Titanium dioxide pigments have the highest refractive index of all white colorants, so they are suitable for lightening colours. Of the three known crystal modifications of titanium dioxide, Brookit has no role in the production of tattoo inks, and because of its strong photochemical activity, Anatas has only a very little role in the production of tattoo inks. As shown in figure 6 , the most important crystal modification of titanium dioxide for tattoo inks is Rutil [5].

\section{Iron Oxide Pigments}

Many variants of iron oxide are used as yellow-, red- and brown-hued pigments. The characteristics of iron oxides limits their use in tattoo inks but also makes them suited for permanent make- up. Iron oxides have a dull and non-brilliant hue compared to inorganic pigments. Iron oxide pigments have a very high opacity. Figure 7 shows that, in contrast to iron oxide red, which is heat stable to temperatures up to a $1,000^{\circ} \mathrm{C}$, yellow and brown iron oxides are not heat stable. In general, tattoos made of iron oxide pigments tend to change to a red hue over time. The reason for this is the tendency of iron oxide to change to the heat-stable red haematite.

\section{Chromium Oxide Green}

The chromium oxide pigments chromium oxide green $\left(\mathrm{Cr}_{2} \mathrm{O}_{3}\right)$ and chromium oxide hydrate green (viridian) $\left(\mathrm{Cr}_{2} \mathrm{O}_{3} \times 3 \mathrm{H}_{2} \mathrm{O}\right)$ are chromatically limited to green. Their authentic characteristics and their heat stability are comparable to iron oxide pigments. During production, hexavalent chromium is reduced to trivalent chromium. Chromium oxides must be closely monitored for soluble chromium VI [5]. These pigments are only used in permanent make-up due to their dull and 'dirty' hue.

\section{Impurities of Inorganic Pigments}

As mentioned before, inorganic pigments always have unwanted and quality-diminishing impurities. Nickel, chromium, copper and cobalt are 


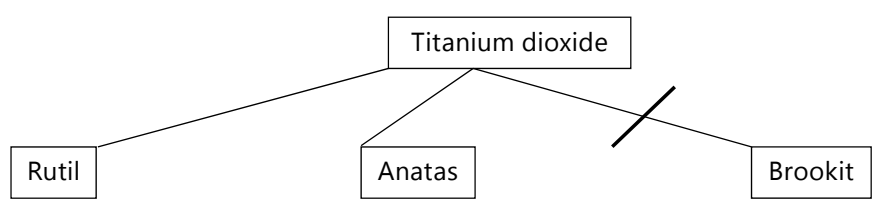

Chemically inert pigment More bluish than anatas Low photochemical activity Refractive index: 2.75

More yellowish than Rutil Pronounced photochemical activity

Refractive index: 2.5

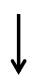

Suitable for tattoo colours
Unsuitable for tattoo colours

Initiate photochemical degradation of pigments in connection with UV light

Fig. 6. The crystal modifications of titanium dioxide.

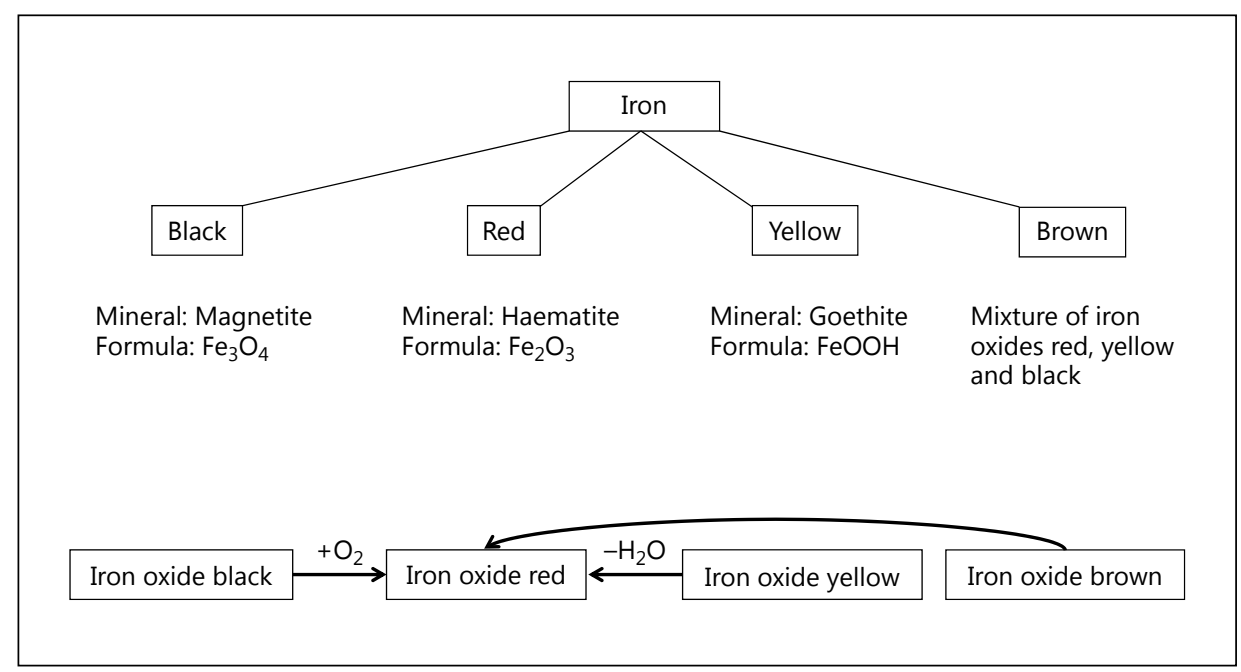

Fig. 7. Variants of iron oxide pigments.

commonly found in the analyses of iron oxide pigments, but these do not lead to allergic reactions as often as expected. Perhaps the reason is the encasing of these different metals in the spinel structure. Further research is required on this matter.

\section{Organic Pigments}

The use of organic pigments enables us to imitate almost every colour the human eye can perceive. In comparison to inorganic pigments, organic pigments are more brilliant and have high- 


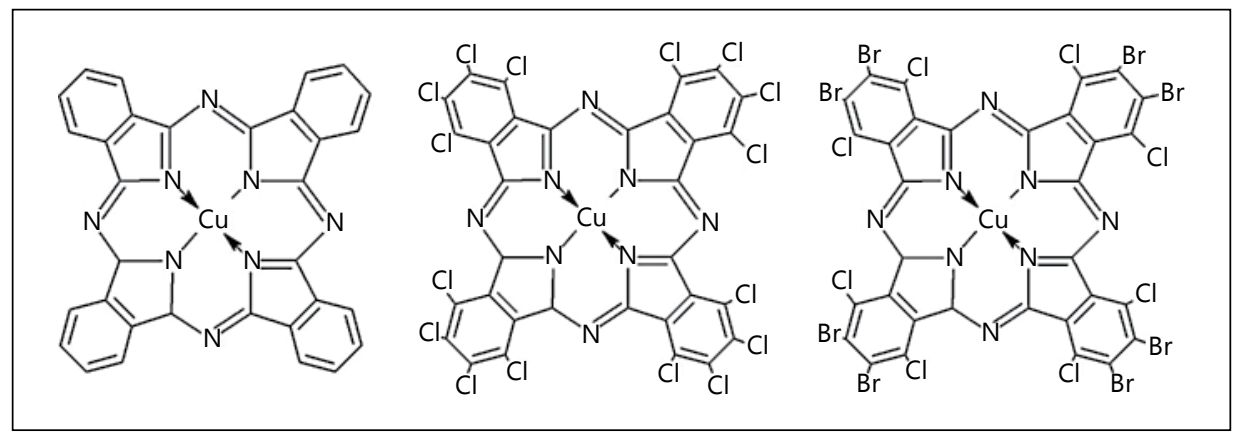

Fig. 8. The polycyclic pigments $\mathrm{Cl} 74160, \mathrm{Cl} 74260$ \& Cl 74265.

er colour strength when blended with white. The variants of these pigments cover the whole colour spectrum but have worse dispersibility than inorganic pigments. Organic pigments can be roughly divided into azo and polycyclic pigments.

\section{Azo Pigments}

Azo pigments are compounds that contain the azo group $-\mathrm{N}=\mathrm{N}$ - chained to $\mathrm{sp} 2$-hybridised $\mathrm{C}$ atoms. The generic formula is

$$
\begin{aligned}
& \text { Aromatic }-\mathrm{N} \searrow \mathrm{N}-\mathrm{R} \\
& \mathrm{R}=\text { rest and Aromatic }=\text { aromatic rest. }
\end{aligned}
$$

The amount of azo groups is used to differentiate between mono and diazo compounds. Compounds with three or more azo groups are not used in any technical scenario. The most-used manufacturing process for these pigments is the azo coupling reaction $[6,7]$.

\section{Polycyclic Pigments}

Polycyclic pigments are not a uniform class of pigments; they are a round-up of all non-azo pigments. With the exception of triphenylmethane pigment, all polycyclic pigments have ring sys- tems. A special case is the polycyclic metal complex of the phthalocyanine system, as shown in figure 8 .

\section{Impurities in Organic Pigments}

Due to their nature, organic pigments have fewer impurities than inorganic pigments. The contamination with heavy metals is much lower compared to inorganic pigments, but due to their production process, they can contain aromatic amines as unwanted accompanying substances. Analytical findings of heavy metals like copper can be traced back to copper bound to phthalocyanine pigments.

\section{Carbon Black}

Soot, also called Carbon Black, is one of the pigments most suited for tattoo ink. Depending on the manufacturing process and curing, these pigments are very different in size and therefore are different in their dispersibilities. Often, the end prodcut is described to have an aerodynamic diameter of 0.1 micrometer. The origins for manufacturing are small spherical parts (the so-called primary particles or nodules), with a size of 15-300 nm. These particles are melted to particle aggregates with an aerodynamic diameter of $85-500 \mathrm{~nm}$. As shown in figure 9 , strong electric forces keep these aggregates 


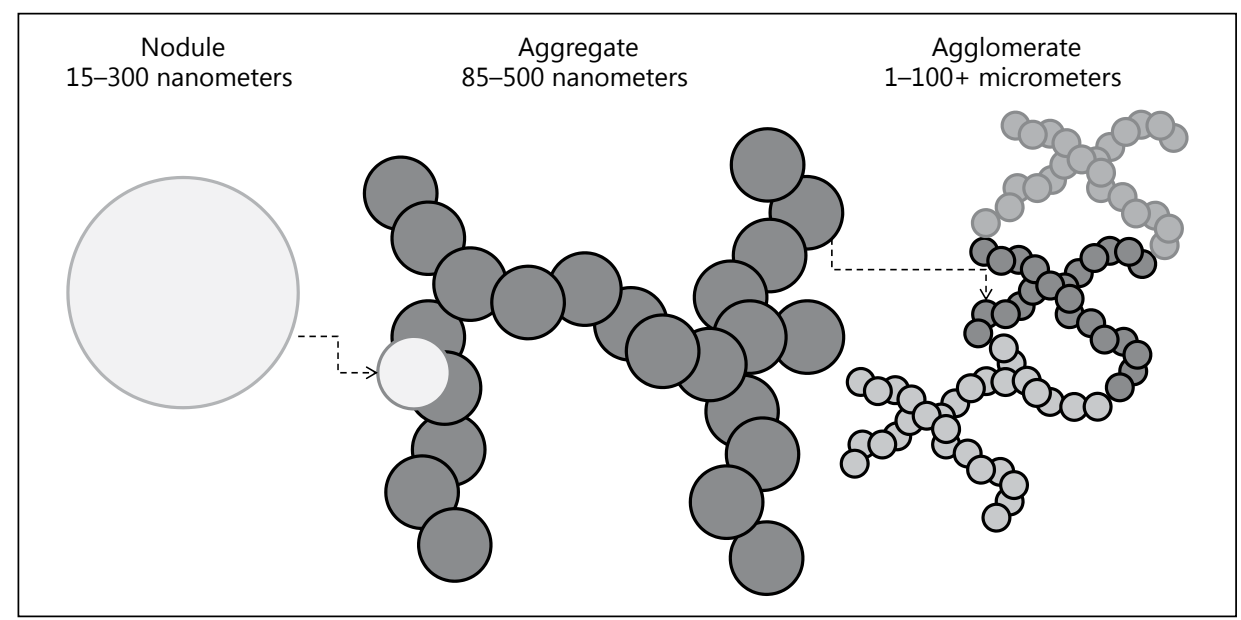

Fig. 9. Structural development of Carbon Black.

together, and when associated with other aggregates, they form agglomerates. All of this happens during the manufacturing process, and consequently, the majority of Carbon Black on the market consists of agglomerates sized 1-100 $\mu \mathrm{m}$ because these agglomerates, once formed, never break. For better handling and to minimise the occurrence of dust, Carbon Black is sold in pellets with a size of $0.1-1 \mathrm{~mm}$. So, the ultrafine primary particles are only found in the production furnace [8].

\section{Impurities of Carbon Black}

Depending on the manufacturing process, Carbon Black can contain impurities in the form of PAHs.

\section{Filler}

Fillers usually consist of inorganic substances with different chemical composition and physical properties. Their main task is to fill the tattoo ink with spatial framework material to influence storage properties. The most-used fillers in tattoo inks are silica and barium sulphate.

\section{Barium Sulphate}

There are two different types of barium sulphate: the naturally occurring, so-called baryt and the synthetically manufactured blanc fixe. The latter is free of unwanted accompanying substances and matches the strict legislative regulation regarding the solubility of barium [5]. As shown in figure 10, barium sulphate is used as a flocculation partner for organic pigments. With controlled flocculation of organic pigments with barium sulphate, the properties relevant for manufacturing, for example, dispersibility, are optimised. The sediment that develops during longer storage is redispersed better, and no demixing of pigments with different densities occurs.

\section{Measurements Taken by the Manufacturer to Ensure Consumer Safety and Satisfaction}

To ensure a high standard of quality, there are two dimensions. One dimension is consistency between each batch of a certain colour, i.e., every batch of a dark green colour is the same shade of dark green. To achieve this goal, standard quality 
Fig. 10. Controlled flocculation (simplified, not to scale).

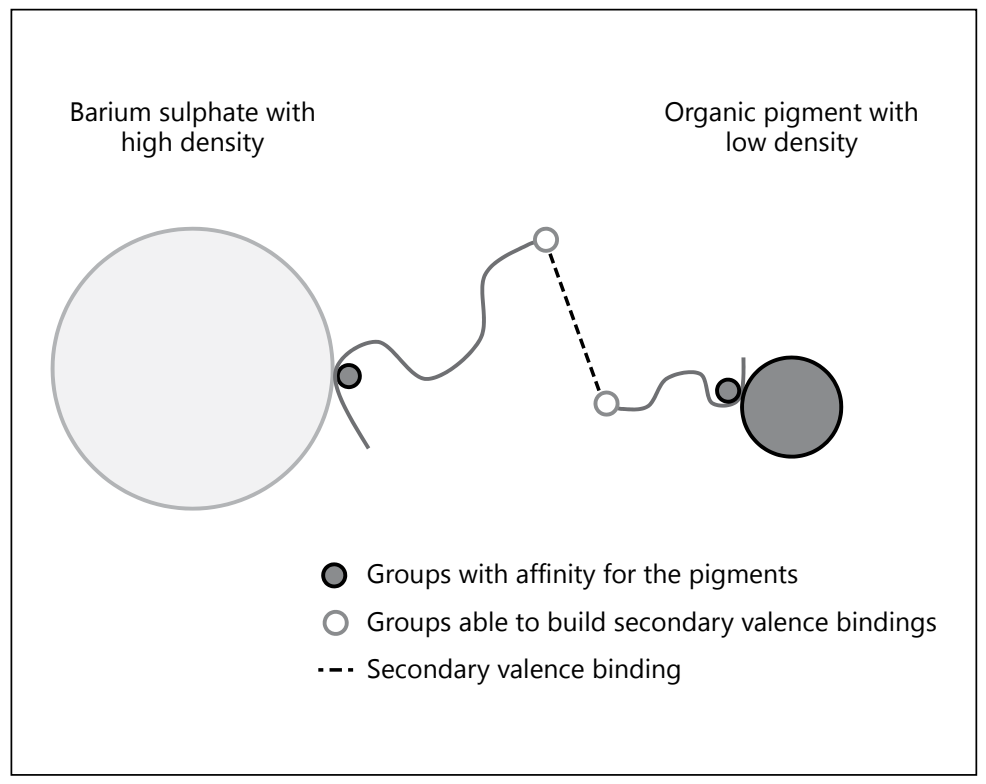

control processes are enforced as in every other colour-processing or colour-producing industry. The other dimension is safety. Every batch of raw materials is tested for inorganic impurities, like heavy metals, or organic impurities, like PAHs. During production, the accidental intake of nickel is prevented using only tools that have either no or very little nickel contamination. Also, all things made out of plastic that directly contact tattoo inks have to conform to the strict criteria of the numerous EU and FDA regulations. Every batch is sterilised with non-ionising gamma rays to ensure that they contain no microbiological pollution. Also, random samples are taken after sterilisation for microbiological testing to ensure that the sterilisation has worked as intended, and all pieces of the packaging are chosen with the aforementioned EU and FDA regulations in mind. Of course, all of these steps are documented to ensure traceability, which is very important, not only as a tool for guaranteeing consumer safety, but also as a tool to facilitate continuous improvement in manufacturing methods.

\section{Conclusion and Outlook}

The manufacturing of tattoo inks with better safety depends on the legislation. A general prohibition of pigments or limitation of pigments with reference to cosmetic ordinances is not suitable. As a result, pigments that have been in use for decades and are much safer than pigments that have not undergone cosmetic safety evaluations have been banned. Also, the approach for using pigments that are regulated by the ordinance for cosmetics and are allowed for use in every product is wrong because many of the allowed pigments are azo pigments and are therefore contaminated with aromatic amines. Instead of a regulation on pigments, the introduction of security dossiers for tattoo colours with no-observed-adverse-effect level and acceptable daily intake values would improve the situation. But, the scientific data needed to calculate these values is inadequate. A standardised EU legislation with validated methods of examination is desirable and would be useful. 


\section{References}

1 DIN EN 971-1:1996-09, Paints and Varnishes - Terms and Definitions for Coating Materials - Part 1. Berlin, Beuth, 1996.

2 Nanetti P: Lackrohstoffkunde. Hannover, Vincentz, 1997.

3 Umbach W: Kosmetik und Hygiene. Weinheim, Wiley-VCH, 2004.
4 DIN 55943-11-1993, Colouring Materials; Terms and Definitions. Berlin, Beuth, 1993.

5 Goldschmidt A, Streitberger HJ: BASFHandbuch Lackiertechnik. Hannover, Vincentz, 2002.
6 Zollinger H: Chemie der Azofarbstoffe. Basel, Birkhäuser, 1958.

7 Zollinger H: Diazo Chemistry. Weinheim, VCH-Verlagsgesellschaft, 1994.

8 International Carbon Black Association (ICBA): Carbon Black User's Guide Safety, Health, \& Environmental Information. ICBA, 2004

Michael Dirks, Dipl.-Ing. (FH)

H-A-N GmbH

Eberhard Bauer Strasse 32

DE-73734 Esslingen (Germany)

E-Mail m.dirks@h-a-n.de 